\section{Dialogue: Vitamin D, statins and atherosclerotic progression in paediatric lupus}

\author{
Gary Gilkeson, ${ }^{1}$ Sue Manzi ${ }^{2}$
}

To cite: Gilkeson G, Manzi S. Dialogue: Vitamin D, statins and atherosclerotic progression in paediatric lupus. Lupus Science \& Medicine 2015;2: e000081. doi:10.1136/lupus2015-000081

Received 2 January 2015 Accepted 6 January 2015

\section{SLinked}

http://dx.doi.org/10.1136/ lupus-2014-000037

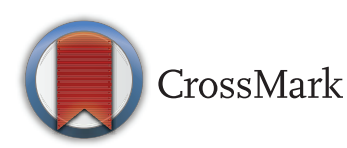

1Department of Medicine, Medical University of South Carolina and the Medical Research Service, Ralph H. Johnson, VAMC, Charleston, South Carolina, USA

${ }^{2}$ Department of Medicine, Allegheny Health Network, and Professor Medicine Temple University School of Medicine, Pittsburgh, Pennsylvania, USA

Correspondence to Gary S Gilkeson; gilkeson@musc.edu
In the last six months, Lupus Science and Medicine has published two papers by Robinson $e t a l^{12}$ that were secondary analyses and substudies of the Atherosclerosis Prevention in Pediatric Lupus Erythematosus (APPLE) study. The major emphasis of the two papers was (1) the association of vitamin D levels at baseline with inflammatory markers, ethnicity, season, lipid status and disease damage in the first paper, (2) and with progression of carotid intimal thickening in patients treated with atorvastatin in the second paper. The original APPLE study was designed to evaluate whether statin therapy (atorvastatin) could affect atherosclerosis progression over a 3-year period in paediatric patients without hypercholesterolaemia. ${ }^{3}$ The significance of this study is obvious, given the accelerated atherosclerosis reported in patients with lupus. There was no statistically significant effect of atorvastatin on carotid intimal thickening progression. Studying underlying mechanisms of this proatherosclerotic effect of lupus in paediatric patients devoid of many of the confounding risk factors present in adults with lupus provides an opportunity to assign causation and response to treatment. The Lupus Atherosclerosis Prevention Study ${ }^{4}$ assessed the effect of statins on atherosclerotic disease progression in 200 adults with no clinical evidence of vascular disease and without elevated cholesterol levels. Like the APPLE study, statin therapy had no significant impact on atherosclerotic progression in adults with lupus. The studies published in Lupus Science and Medicine addressed whether vitamin D status at baseline affected progression of carotid atherosclerosis.

Vitamin D is reported to have myriad physiological effects. A number of published reports, primarily retrospective analyses, suggest a role for vitamin $\mathrm{D}$ in bone health, atherosclerosis, efficacy of statin therapy, cancer and autoimmune diseases. Investigators have reported lower vitamin $\mathrm{D}$ in patients with lupus versus non-lupus controls. In addition, low vitamin D levels have been associated with more lupus disease flares and end organ damage. ${ }^{5}$ These combined observations provided the biological rationale for examining whether vitamin D levels affected the results of the APPLE trial.

In the first paper, the authors determined the variables associated with vitamin $\mathrm{D}$ status at baseline. ${ }^{2}$ Perhaps not surprisingly, over $90 \%$ of participants in the trial had vitamin $\mathrm{D}$ insufficiency $(25(\mathrm{OH}) \mathrm{D}<30 \mathrm{ng} / \mathrm{mL}$ ), whereas $30 \%$ were vitamin D deficient $(<20 \mathrm{ng} / \mathrm{mL})$ and $10 \%$ were severely deficient $(<10 \mathrm{ng} / \mathrm{mL})$. The participants were divided into two groups: $<20$ and $>20 \mathrm{ng} / \mathrm{mL}$ of $25(\mathrm{OH}) \mathrm{D}$ for comparison ( $n=61$ vs $n=140$, respectively). As expected, there was a correlation between the serum vitamin $\mathrm{D}$ levels and the season in which the blood was drawn. There was also a significant correlation with ethnicity, with AfricanAmericans having the highest percentage of vitamin D deficiency. Other 'lupus' factors that correlated with vitamin $\mathrm{D}$ deficiency at baseline were longer disease duration and more organ damage as measured by the Systemic Lupus International Cooperating Clinics (SLICC) damage index. The primary finding of the paper was that low vitamin D levels at baseline were associated with a high sensitivity $\mathrm{C}$ reactive protein (hsCRP) (2.2 in the vitamin D-insufficient group vs 4.6 in the vitamin D-deficient group). Higher low-density lipoprotein cholesterol levels were also associated with vitamin $\mathrm{D}$ insufficiency. In a multivariate analysis controlling for other variables, hsCRP remained significantly associated with vitamin D status.

The primary limitation of this study was that the associations between vitamin $\mathrm{D}$ status and baseline variables did not include baseline carotid intima-media thickness (CIMT), a measure of atherosclerosis. Thus, the second paper by the same authors was of greater interest since this study examined the interaction between vitamin D deficiency (serum $25(\mathrm{OH})$ 
$\mathrm{D}<20 \mathrm{ng} / \mathrm{mL}$ ) at baseline and atorvastatin or placebo treatment and CIMT progression at 3 years adjusting for other key risk factors. ${ }^{1}$

The authors reported that participants who had serum $25(\mathrm{OH}) \mathrm{D}$ levels $>20 \mathrm{ng} / \mathrm{mL}$ and were treated with atorvastatin had significantly less progression of CIMT. The interaction between vitamin D status and atorvastatin treatment and CIMT progression appeared to be independent of a decrease in lipid levels or hsCRP. Participants whose vitamin D levels increased during the study from deficient to insufficient or sufficient also showed a trend towards less progression of CIMT, but it was not significant. Vitamin D measures were not associated with changes in lipid levels, hsCRP or lupus activity scores. The authors propose a potential hypothesis of a direct interaction of vitamin $\mathrm{D}$ status and atorvastatin in preventing progression of atherosclerosis. They suggest that this hypothesis be further tested in longitudinal studies in adult and paediatric patients with lupus.

The reviewers felt that there were many strengths to the study including the parent APPLE trial, which was a well-conducted randomised, multicentre, placebo controlled trial with clearly defined and centralised measurements of vitamin $\mathrm{D}$ and other risk factors as well as outcomes (CIMT). There is a biological rationale to study vitamin $\mathrm{D}$ and statins in atherosclerosis development and progression. The second study provides a longitudinal analysis of vitamin D levels, lipid levels, lupus activity and CIMT over 3 years. The authors appropriately acknowledged the limitations of secondary analyses and did not overstate the results. They considered these findings to be hypothesis generating rather than definitive proof.

As with any good study, often more questions are raised than are answered. One concern is that almost all the patients were vitamin D insufficient with $30 \%$ vitamin $\mathrm{D}$ deficient. Thus, there is really no comparator group of individuals with sufficient vitamin D levels. Although there was a trend towards less CIMT progression over time in those whose vitamin $\mathrm{D}$ levels rose during the study, this did not reach significance due to the small numbers. It was unclear from this study whether replacement of vitamin D to $>30 \mathrm{ng} / \mathrm{mL}$ had an impact on disease progression. It is also unknown whether there is a time lag between achieving 'normal' vitamin $\mathrm{D}$ levels and beneficial biological effects. The reviewers also noted that vitamin $\mathrm{D}$ levels were associated with CIMT at baseline. Since baseline CIMT is the strongest predictor of progression of CIMT, this variable should have been controlled for in the analysis. Other questions were related to ethnicity since AfricanAmericans generally have lower vitamin D levels as well as greater CIMT than Caucasians. If one removes ethnicity, does the vitamin D effect still remain significant? There was no systematic tracking of vitamin D supplementation and it is stated that the atorvastatin group took more 'multivitamins' than did the control group. Could differences in vitamin supplementation play a role in the observations made in this study?

In summary, these two studies provide interesting observations regarding vitamin $\mathrm{D}$, statins and atherosclerosis/CIMT. The mechanisms for a vitamin D amplification of statin effect requires further study to test this hypothesis. The participants with higher vitamin D levels did not have lower cholesterol, decreased disease activity or lower hsCRP levels. Vitamin D may modify a variety of biological pathways that could affect atherosclerosis and effectiveness of statins. Although this observation has potential clinical relevance, the wide spread screening for vitamin $\mathrm{D}$ deficiency and the increasing use of vitamin D supplementation may make it difficult to study the effects of vitamin deficiency in the future.

Competing interests None.

Provenance and peer review Commissioned; internally peer reviewed

Open Access This is an Open Access article distributed in accordance with the Creative Commons Attribution Non Commercial (CC BY-NC 4.0) license, which permits others to distribute, remix, adapt, build upon this work noncommercially, and license their derivative works on different terms, provided the original work is properly cited and the use is non-commercial. See: http:// creativecommons.org/licenses/by-nc/4.0/

\section{REFERENCES}

1. Robinson $A B$, Tangpricha $\mathrm{V}$, Yow $\mathrm{E}$, et al. Vitamin $\mathrm{D}$ status is a determinant of atorvastatin effect on carotid intima medial thickening progression rate in children with lupus: an Atherosclerosis Prevention in Pediatric Lupus Erythematosus (APPLE) substudy. Lupus Sci Med 2014;1:e000037.

2. Robinson $A B$, Tangpricha V, Yow E, et al. Vitamin D deficiency is common and associated with increased C-reactive protein in children and young adults with lupus: an Atherosclerosis Prevention in Pediatric Lupus Erythematosus substudy. Lupus Sci Med 2014;1: e000011.

3. Schanberg LE, Sandborg C, Barnhart HX, et al. Premature atherosclerosis in pediatric systemic lupus erythematosus: risk factors for increased carotid intima-media thickness in the atherosclerosis prevention in pediatric lupus erythematosus cohort. Arthritis Rheum 2009;60:1496-507.

4. Petri MA, Kiani AN, Post W, et al. Lupus Atherosclerosis Prevention Study (LAPS). Ann Rheum Dis 2011;70:760-5.

5. Kamen DL. Environmental influences on systemic lupus erythematosus expression. Rheum Dis Clin North Am 2014;40:401-12, vii. 